\title{
Metronidazole Causes Skeletal Muscle Atrophy and Modulates Muscle Chronometabolism
}

\author{
Ravikumar Manickam ${ }^{1}$, Hui Yun Penny Oh ${ }^{1,2}$, Chek Kun Tan ${ }^{1}$, Eeswari Paramalingam ${ }^{1}$ \\ and Walter Wahli 1,3,* (D) \\ 1 Lee Kong Chian School of Medicine, Nanyang Technological University, 11 Mandalay Road, \\ Singapore 308232, Singapore; ravikumar_vet@hotmail.com (R.M.); HOH001@e.ntu.edu.sg (H.Y.P.O.); \\ willcktan@hotmail.com (C.K.T.); eeswari28@hotmail.com (P.E.) \\ 2 Interdisciplinary Graduate School, NTU Institute for Health Technologies, Nanyang Technological \\ University, 50 Nanyang Avenue, Singapore 639798, Singapore \\ 3 Center for Integrative Genomics, University of Lausanne, Le Génopode, CH-1015 Lausanne, Switzerland \\ * Correspondence: walter.wahli@ntu.edu.sg; Tel.: +65-6904-7012
}

Received: 24 July 2018; Accepted: 14 August 2018; Published: 16 August 2018

\begin{abstract}
Antibiotics lead to increased susceptibility to colonization by pathogenic organisms, with different effects on the host-microbiota relationship. Here, we show that metronidazole treatment of specific pathogen-free (SPF) mice results in a significant increase of the bacterial phylum Proteobacteria in fecal pellets. Furthermore, metronidazole in SPF mice decreases hind limb muscle weight and results in smaller fibers in the tibialis anterior muscle. In the gastrocnemius muscle, metronidazole causes upregulation of Hdac4, myogenin, MuRF1, and atrogin1, which are implicated in skeletal muscle neurogenic atrophy. Metronidazole in SPF mice also upregulates skeletal muscle FoxO3, described as involved in apoptosis and muscle regeneration. Of note, alteration of the gut microbiota results in increased expression of the muscle core clock and effector genes Cry2, Ror- $\beta$, and E4BP4. PPAR $\gamma$ and one of its important target genes, adiponectin, are also upregulated by metronidazole. Metronidazole in germ-free (GF) mice increases the expression of other core clock genes, such as Bmal1 and Per2, as well as the metabolic regulators FoxO1 and Pdk4, suggesting a microbiota-independent pharmacologic effect. In conclusion, metronidazole in SPF mice results in skeletal muscle atrophy and changes the expression of genes involved in the muscle peripheral circadian rhythm machinery and metabolic regulation.
\end{abstract}

Keywords: metronidazole; gut dysbiosis; skeletal muscle atrophy; circadian rhythm

\section{Introduction}

The mammalian gut hosts the most diverse population of microorganisms, including bacteria, archaea, protozoa and fungi, and even viruses. The gut microbiota primarily provides protection against pathogenic microorganisms and inflammation and helps with digestion and energy metabolism by modulating various host signaling pathways [1]. The daily rhythmicity of the gut microbiota and its metabolites modulates the host circadian rhythm [2]. Furthermore, gut dysbiosis and circadian rhythm disruption are associated with metabolic syndrome and diseases such as obesity, type 2 diabetes, and inflammatory bowel disease [3].

The gut microbiota composition is modulated by diet and antibiotics [4,5]. Antibiotics alter microbiota composition and expression of microbial genes and derived metabolites such as bile acid products and short-chain fatty acids, methylamines, and indoles [4,6]. Alteration of gut microbiota composition may lead to deleterious effects on the host and colonization by antibiotic-resistant pathogens [7]. Most of these findings come primarily from studies in animals exposed to various 
antibiotics, including metronidazole (1-(2-hydroxyethyl)-2-methyl-5-nitroimidazle) for a period of 4 weeks [8,9].

Furthermore, comparative analyses of specific pathogen-free (SPF) and germ-free (GF) mice are often used to study the effects of microbiota on host metabolic and immune functions. Several studies have demonstrated that GF mice are protected from diet-induced obesity, low-grade inflammation, and glucose intolerance as compared to conventionally raised animals $[10,11]$. Furthermore, microbiota transfer via fecal transplantation evokes modifications in body weight and insulin sensitivity in both rodents and humans $[4,10,12]$.

Metronidazole is in the nitroimidazole class of antimicrobial drugs, frequently used to treat anaerobic, protozoal, and Helicobacter pylori infections [13]. It causes transient loss of colonization resistance to pathogens [14]. When administered orally, metronidazole is absorbed almost completely and distributed widely within the body. It is extensively metabolized by the liver, most likely by the hepatic cytochrome P450 system, and its degradation products are excreted in urine and feces [13]. Metronidazole is believed to enter the cerebrospinal fluid and the central nervous system, which can cause neurotoxicity [13]. The effects in the central nervous system range from seizures to metronidazole-induced encephalopathy and cerebellar syndrome. Recently, antibiotic-associated encephalopathy was identified as a cause of delirium in patients receiving antibiotics including metronidazole; however, peripheral neuropathy is the most common adverse effect of metronidazole [15]. Of interest, many muscle functions, including energy metabolism, myokine secretion, tissue homeostasis, and regeneration, are influenced by the skeletal muscle peripheral clock and the clock-controlled genes [16-18], but the mechanism by which metronidazole causes muscle weakness is not known. Because the microbiota participates in the control of the liver peripheral clock [19], we hypothesized that, among other effects, metronidazole may also influence the skeletal muscle peripheral clock.

Here we demonstrate that metronidazole causes changes in the expression of genes implicated in the muscle peripheral clock machinery, metabolic regulators, and skeletal muscle neurogenic atrophy that might be associated with peripheral neuropathy, the most commonly observed adverse side effect. Metronidazole also causes protein degradation and thus acts as a double-edged sword in skeletal muscle atrophy.

\section{Results}

\subsection{Metronidazole Enhances Susceptibility to Colonization by Proteobacteria and Enrichment} of Erysipelotrichales

Consistent with other publications $[8,9]$, we found that metronidazole treatment had a marginal impact on the major bacterial phyla distribution in specific pathogen-free (SPF) mouse fecal pellets analyzed by $16 \mathrm{~S}$ rRNA gene sequencing. However, we found a slight but significant increase in the susceptibility to colonization by Proteobacteria, especially Parasutterella AJ308395_s, compared to nontreated control mice (Figure 1a). Furthermore, there was substantial enrichment of the bacterial species Erysipelotrichales EF603943_s in the metronidazole-treated SPF mice when compared to the nontreated control mice (Figure 1b). 
(a)

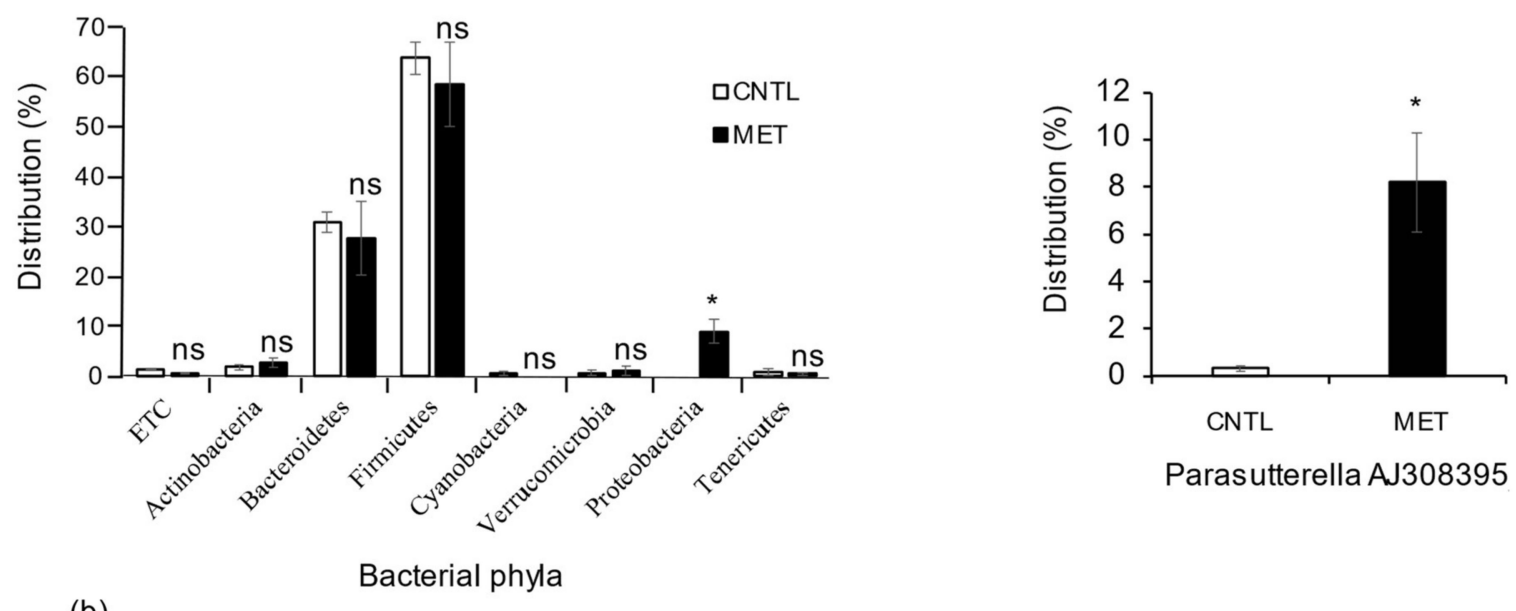

(b)

Heat map (Gradient)

Min. Max.

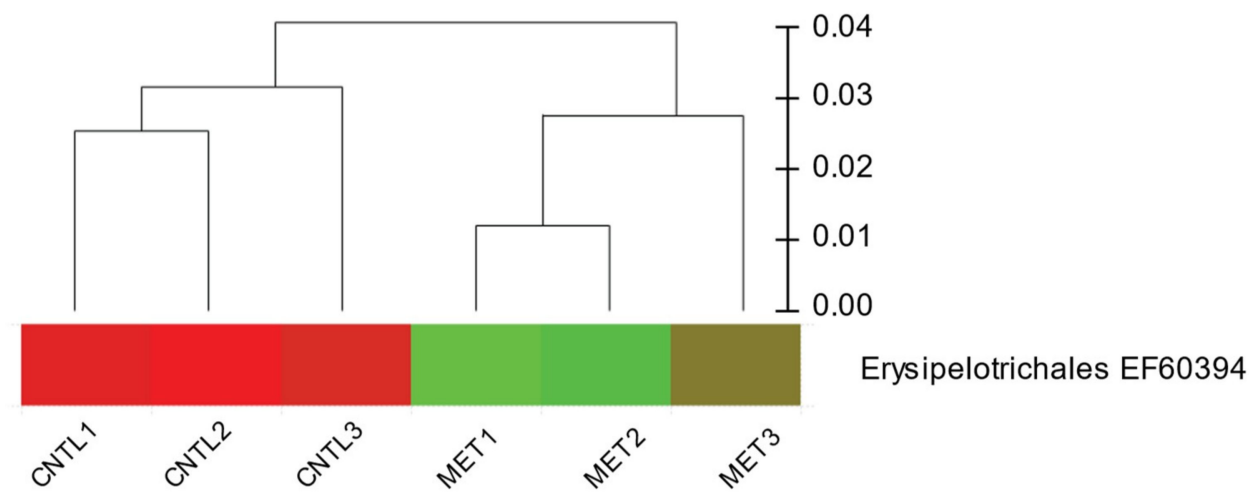

Figure 1. Metronidazole enhances susceptibility to colonization by Proteobacteria and enrichment of Erysipelotrichales. 16s rRNA genes were sequenced from DNA extracted from the fecal pellets of metronidazole (MET)-treated and nontreated control (CNTL) specific pathogen-free (SPF) mice. (a) Percentage representation of the bacterial phyla distribution in fecal pellets of metronidazole-treated and nontreated control SPF mice demonstrating increased susceptibility to colonization by Proteobacteria, especially Parasutterella. $N=3$ mice per group. Data are presented as means \pm standard error of the mean (SEM). Asterisks indicate statistically significant differences $(*, p<0.05 ; \mathrm{ns}$, nonsignificant with student's $t$ test). (b) Gradient heatmap showing enrichment of the bacterial species Erysipelotrichales in metronidazole-treated SPF mice as compared to nontreated controls. Relative abundance of the bacterial species is color coded from minimum (red) to maximum (green). $N=3$ mice per nontreated control (CNTL1-3) and metronidazole-treated (MET1-3) groups.

\subsection{Metronidazole Causes Skeletal Muscle Atrophy}

The body weight of metronidazole-treated SPF mice was marginally but not significantly reduced when compared to nontreated control mice (Figure 2a). However, the body weight of metronidazole-treated germ-free (GF) mice was significantly reduced when compared to nontreated control mice (Supplementary Figure S1a). The weight of the hind limb muscles of metronidazole-treated SPF and GF mice was significantly reduced compared to nontreated control mice, with the exception of the soleus in GF mice (Figure $2 b$ and Supplementary Figure S1b). Noticeably, the relative weight of the hind limb muscles of both SPF and GF mice did not display 
any significant differences between metronidazole-treated and nontreated control mice (data not shown). However, histological examination of the tibialis anterior muscle at the mid-belly region of the metronidazole-treated SPF mice revealed a significant decrease in larger myofibers and a concomitant increase in smaller myofibers compared to nontreated control mice (Figure 2c). Taken together, these data suggest that metronidazole treatment might lead to skeletal muscle atrophy.

(a)

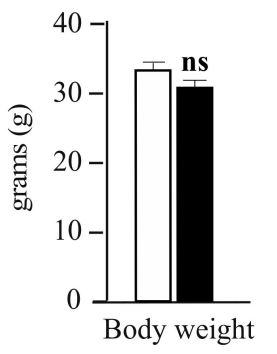

(b)



(c)

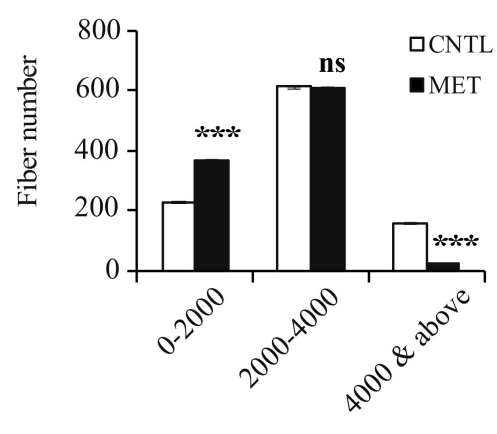

Myofiber area $\left(\mu \mathrm{m}^{2}\right)$

Figure 2. Metronidazole causes reduced hind limb muscle weight and myofiber surface area. (a) Metronidazole treatment of SPF mice resulted in a marginal, nonsignificant decrease in body weight compared to nontreated controls. $N=5$ mice per group. (b) Absolute hind limb muscle weights of tibialis anterior (TA), extensor digitorum longus (EDL), gastrocnemius (GAS), soleus (SOL), and quadriceps (QUAD) muscles of metronidazole-treated SPF mice compared to nontreated controls. $N=5$ mice per group. (c) Myofiber numbers according to myofiber surface area in the tibialis anterior in metronidazole-treated and nontreated SPF mice. $N=3$ mice per group. A thousand random myofibers per mouse were counted across the mid-belly region of the tibialis anterior muscle cryosections. Data presented as means \pm SEM of 3000 myofibers per group (CNTL and MET). Asterisks indicate statistically significant differences $\left({ }^{*}, p<0.05 ;{ }^{* *}, p<0.01 ;{ }^{* *}, p<0.001\right.$; ns, nonsignificant with student's $t$ test).

\subsection{Metronidazole Causes Skeletal Muscle Neurogenic Atrophy}

Denervation of skeletal muscle upregulates the histone deacetylases 4 and 5 (Hdac4/5), which then repress dachshund family transcription factor 2 (Dach2), a negative regulator of myogenin. Increased myogenin in turn upregulates the ubiquitin E3 ligases MuRF1 and atrogin1, causing skeletal muscle atrophy [20]. Our analysis of gene expression in the mouse gastrocnemius revealed a significant upregulation of skeletal muscle neurogenic atrophy markers, such as Hdac4, myogenin, MuRF1, and atrogin 1 expression, in metronidazole-treated SPF mice as compared to nontreated control animals (Figure 3a). This effect was not observed in GF mice treated with metronidazole (Figure 3b). The expression level of Hdac5 did not differ in SPF mice treated with and without metronidazole (Supplementary Figure S2a). Dach2 expression level was reduced in metronidazole-treated SPF mice when compared to nontreated control mice (Supplementary Figure S2a), but this trend did not reach statistical significance $(p=0.063)$.

Forkhead box protein $\mathrm{O} 3$ (FoxO3) is a key transcription factor in protein breakdown, because it modulates the activity of several actors in the ubiquitin-proteasome and autophagy-lysosomal proteolytic pathways, including mitochondrial autophagy, also known as mitophagy [21,22]. Analysis of mouse gastrocnemius revealed a significant increase in FoxO3 expression level in SPF mice treated with metronidazole when compared to nontreated control mice, and the effect was not observed in GF mice (Figure 3a,b). Furthermore, expression levels of the master regulator gene of myogenesis, MyoD, the muscle mass-determining dominant-negative gene myostatin, and the autophagy marker genes Mul1 and LC3-1 were unaffected by metronidazole treatment in SPF mice compared to nontreated 
control mice (Supplementary Figure S2b). Together, these data suggest that metronidazole causes skeletal muscle atrophy through upregulation of myogenin and FoxO3.

(a)

\section{SPF mice}
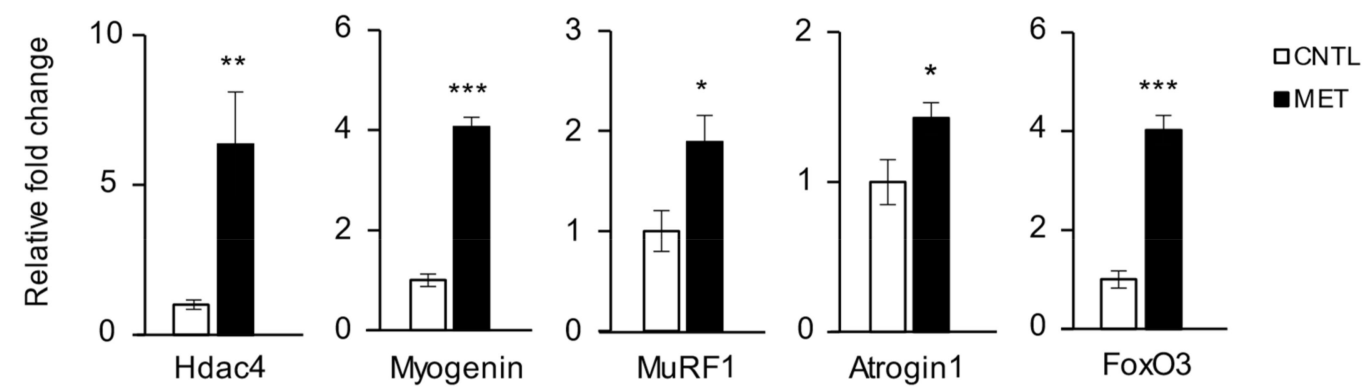

(b)
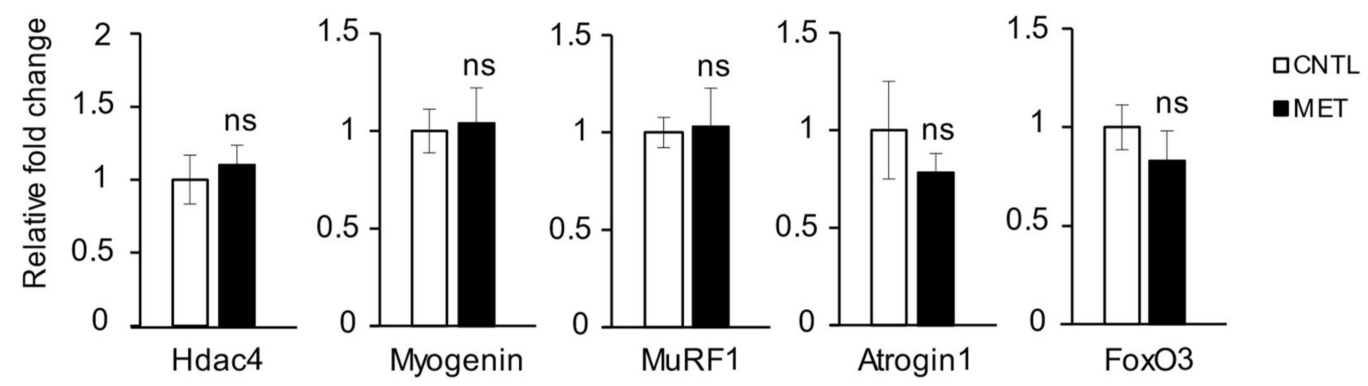

Figure 3. Metronidazole causes changes in the expression of skeletal muscle atrophy genes. Real-time quantitative PCR analysis of Hdac4, myogenin, MuRF1, atrogin1, and FoxO3 expression in gastrocnemius of metronidazole-treated and nontreated (a) SPF and (b) GF mice. $N=5$ mice per group. Data presented as means \pm SEM. Asterisks indicate statistically significant differences $\left({ }^{*}, p<0.05 ;{ }^{* *}, p<0.01\right.$; $* * *, p<0.001 ; \mathrm{ns}$, nonsignificant with student's $t$ test).

\subsection{Metronidazole Is Involved in Skeletal Muscle Energy Metabolism}

FoxO1 is a transcription factor that plays an important role in glucose metabolism by insulin signaling. In skeletal muscle, FoxO1 increases expression of the mitochondrial pyruvate dehydrogenase (acetyl-transferring) kinase isozyme 4 (Pdk4). This kinase phosphorylates subunits of pyruvate dehydrogenase, which inhibits the activity of the enzyme. Inhibition of pyruvate dehydrogenase decreases glucose use and increases fat metabolism, as during fasting [23,24]. We found that FoxO1 expression in gastrocnemius was significantly upregulated in SPF mice treated with metronidazole compared to nontreated control mice (Figure 4a). Of note, metronidazole treatment of GF mice triggered an opposite effect, with a significant reduction in expression level of FoxO1 compared to nontreated control GF mice (Figure $4 \mathrm{~b}$ ). As a FoxO1 target gene, Pdk4 was upregulated in SPF mice treated with metronidazole (not significantly, however, likely because of individual variation) and significantly downregulated, together with FoxO1, in GF mice treated with metronidazole (Figure 4b). Furthermore, there was no change in the expression levels of metabolic regulators such as Sirt1, Ampk, $P g c-1 \alpha, P P A R \alpha$, and PPAR $\beta / \delta$ in SPF mice treated with metronidazole compared to nontreated control animals (Supplementary Figure S2c). 
(a)

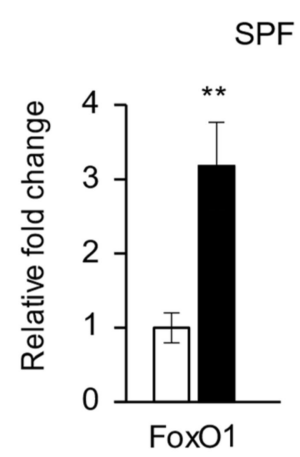

SPF mice

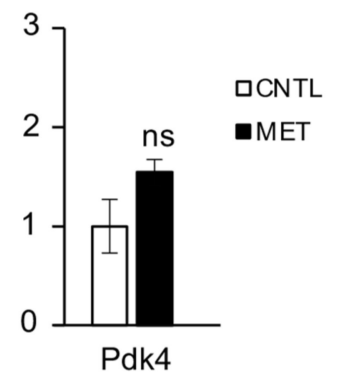

(b)

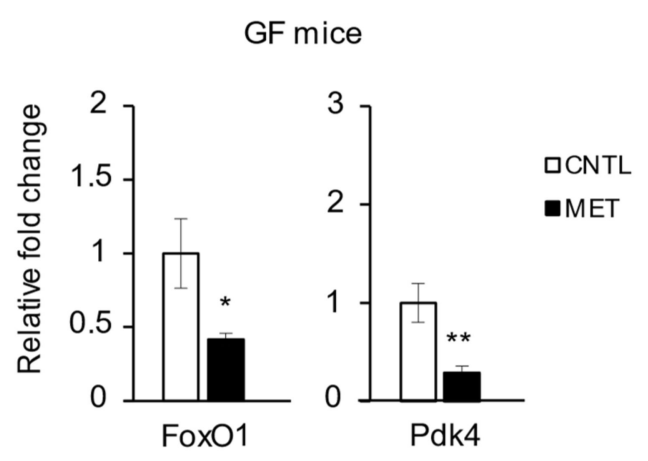

Figure 4. Metronidazole affects the expression of skeletal muscle metabolism genes. Real-time quantitative PCR analysis of FoxO1 and Pdk4 expression in gastrocnemius of metronidazole-treated and nontreated (a) SPF and (b) GF mice. $N=5$ mice per group. Data presented as means \pm SEM. Asterisks indicate statistically significant differences $\left({ }^{*}, p<0.05 ;{ }^{* *}, p<0.01 ; \mathrm{ns}\right.$, nonsignificant with student's $t$ test).

Together, these data suggest that, through its impact on FoxO1 and Pdk4 expression in SPF mice, metronidazole could decrease glucose use in skeletal muscle through an effect on the microbiota, which may participate with other effects in the observed skeletal muscle atrophy. In contrast, in the skeletal muscle of GF mice, metronidazole had a microbiota-independent pharmacologic effect that would promote glucose use.

\subsection{Metronidazole Modulates Expression of Muscle Core Clock and Clock-Controlled Genes}

Transcription of the clock gene Cry1 follows a circadian pattern in skeletal muscle [25], and drugs such as prednisolone and ramipril can modulate skeletal muscle clock genes [26,27]. The gastrocnemius muscles of metronidazole-treated SPF mice displayed significant upregulation of the core clock genes Per2 and Cry2 and a downregulation trend for Bmal1 when compared to nontreated control mice (Figure 5a). Furthermore, Ror- $\beta$ and E4BP4 were also significantly upregulated after metronidazole treatment (Figure 5a). In contrast, the metronidazole-treated GF mice displayed significant upregulation of Bmal1 and downregulation of Per 2 as compared to the nontreated control GF mice, again indicating a microbiota-independent effect opposite to that of the microbiota-dependent effect in SPF mice (Figure 5b). Moreover, expression levels of Clock, Per1, Cry1, Ror- $\alpha$ and Ror- $\gamma$, and Rev-erb $\alpha$ and Rev-erb $\beta$ did not differ significantly, and neither did those of clock-controlled genes $D b p$ and $D e c 2$ in the SPF mice treated with metronidazole (Supplementary Figure S2d).

Of note, PPAR $\gamma$ and its target gene adiponectin $[28,29]$ were significantly upregulated in the SPF mice treated with metronidazole (Figure 6a), an effect that was absent in metronidazole-treated GF mice (Figure $6 b$ ). The expression of other factors important in metabolic regulation, such as Sirt1, $A m p k, P g c-1 \alpha, P P A R \alpha$, and $P P A R \beta / \delta$, was not affected by metronidazole in SPF mice (Supplementary Figure S2c). Together, these data show that in SPF mice, metronidazole affects some, but not all, genes of the muscle clock and genes important in metabolism. In GF mice, metronidazole also affects clock genes, but in a way opposite to that observed in SPF mice. 
(a) SPF mice
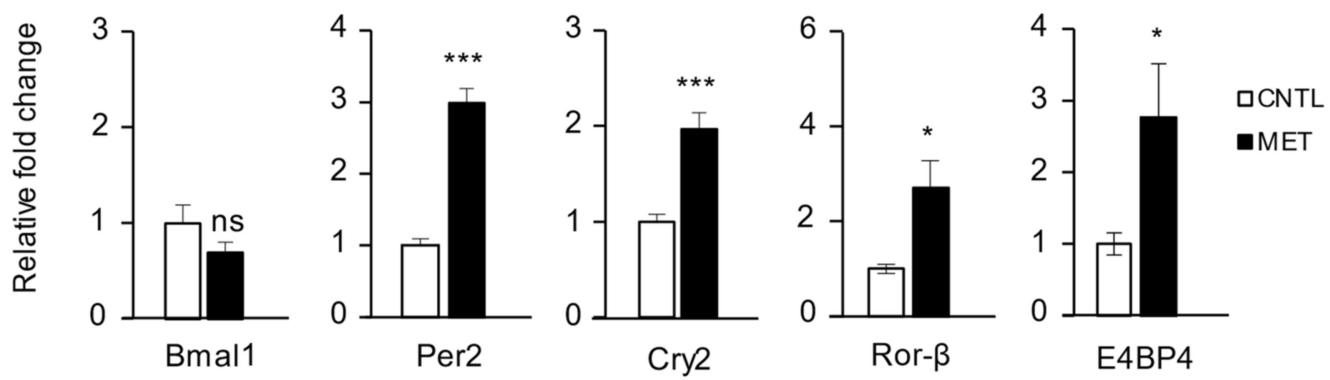

(b)

$$
\text { GF mice }
$$
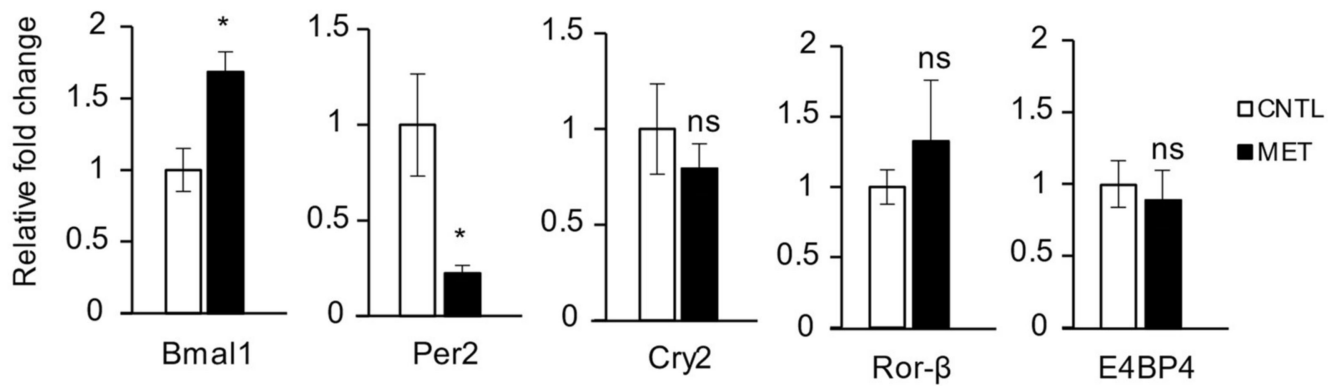

Figure 5. Metronidazole alters skeletal muscle core clock and clock effector gene expression. Real-time quantitative PCR analysis of Bmal1, Per2, Cry2, Ror- $\beta$, and E4BP4 expression in gastrocnemius of metronidazole-treated and nontreated (a) SPF and (b) GF mice. $N=5$ mice per group. Data presented as means \pm SEM. Asterisks indicate statistically significant differences $\left({ }^{*}, p<0.05 ; * * *, p<0.001\right.$; ns, nonsignificant with student's $t$ test).

(a)

SPF mice

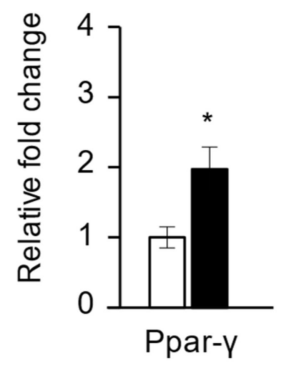

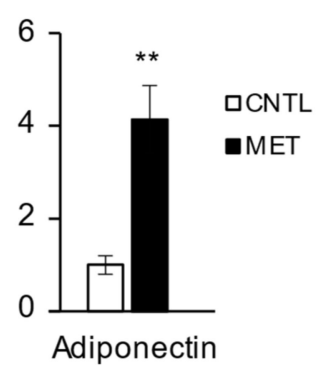

(b)

GF mice

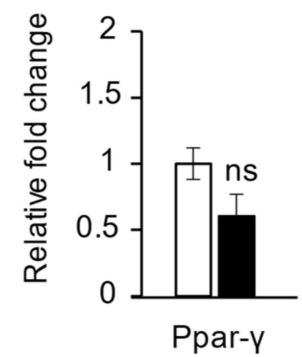

Figure 6. Metronidazole modulates skeletal muscle adiponectin and PPAR $\gamma$ expression. Real-time quantitative PCR analysis of PPAR $\gamma$ and adiponectin expression in gastrocnemius muscle of metronidazole-treated and nontreated (a) SPF (a) and (b) GF mice. $N=5$ mice per group. Data presented as means \pm SEM. Asterisks indicate statistically significant differences $\left(^{*}, p<0.05 ;{ }^{* *}, p<0.01\right.$; ns, nonsignificant with student's $t$ test).

\subsection{Metronidazole Stimulates Expression of RNA Modification of $N^{6}$-Methyladenosine Complex Genes}

RNA modification is now recognized as playing a role in many important biological functions. $\mathrm{N}^{6}$-methyladenosine $\left(\mathrm{m}^{6} \mathrm{~A}\right)$ is an abundant modification of mRNA found in most eukaryotic RNAs [30]. 
$\mathrm{m}^{6} \mathrm{~A}$ can be incorporated by a methyltransferase complex, whose core is a methyltransferase such as 3 (METTL3)/METTL14 heterodimer, in which METTL3 provides the catalytic activity [31]. $\mathrm{m}^{6} \mathrm{~A}$ is recognized by YTH domain-containing proteins, which instruct different complexes to regulate RNA signaling pathways [30]. It was recently found that $\mathrm{m}^{6} \mathrm{~A}$ modification by METTL3 stabilizes MyoD mRNA levels, thereby promoting skeletal muscle differentiation [32]. We therefore analyzed whether metronidazole treatment changes the expression of genes for the methylation complex (Mettl3, Mettl14) and for a YTH domain-recognition protein (Ythdf2), as well as that of the Fto gene, whose mRNA is $\mathrm{m}^{6} \mathrm{~A}$ methylated. We found that Mettl3 and Mettl14 showed increased expression in SPF mice treated with metronidazole compared to nontreated controls (Figure 7a). This effect did not occur in GF mice (Figure 7b). Also, we found no influence of metronidazole on Fto and Ythdf2 expression in SPF mice (Supplementary Figure S2e). Collectively, these results demonstrate that metronidazole affects the expression of genes involved in the RNA epigenetic modification machinery in skeletal muscle.

(a)

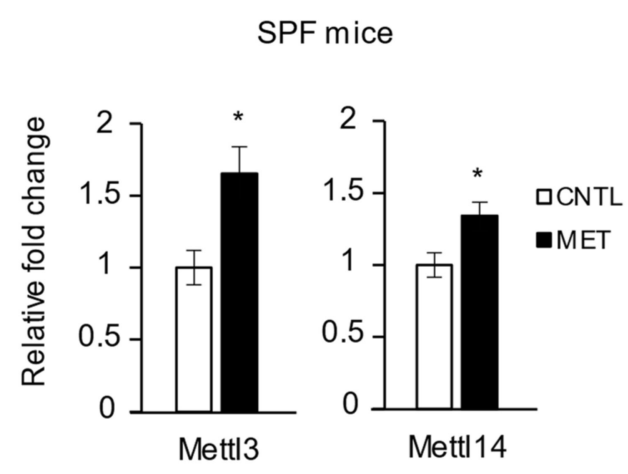

(b)

\section{GF mice}

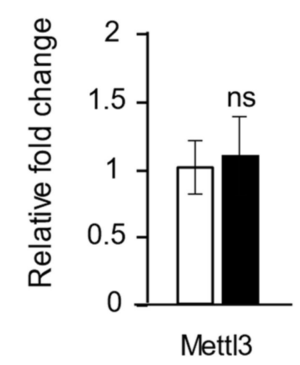

Figure 7. Metronidazole disrupts skeletal muscle RNA epigenetics. Real-time quantitative PCR analysis of RNA $\mathrm{m}^{6} \mathrm{~A}$ methyltransferases Mettl3 and Mettl14 expression in gastrocnemius muscle of metronidazole-treated and nontreated (a) SPF and (b) GF mice. $N=5$ mice per group. Data presented as means \pm SEM. Asterisks indicate statistically significant differences $\left({ }^{*}, p<0.05\right.$; ns, nonsignificant with student's $t$ test).

\section{Discussion}

The goal of this study was to characterize the effects of metronidazole on gut dysbiosis and muscle health. We treated SPF and GF mice for 4 weeks with metronidazole in drinking water, similar to most published studies involving antibiotic-treated mice [8,9]. In this study, all mice had ad libitum access to food and water. We found that metronidazole caused gut dysbiosis with increased susceptibility to colonization by Proteobacteria and enrichment of Erysipelotrichales in the treated SPF mice. A recent report suggested that an increase in Parasutterella is associated with the development and progression of irritable bowel syndrome (IBS) and chronic inflammation of the intestine [33]. Transferring aged gut microbiota to young GF mice led to an increase in Proteobacteria involved in inflammatory aging and inflammation of the small intestine [34]. Similarly, an increase in Erysipelotrichales has been associated with inflammatory aging in older mice when compared to young mice [35]. Here we demonstrate that this dysbiosis correlates with skeletal muscle atrophy with upregulation of Hdac4, myogenin, and FoxO1/O3. It is possible that the increase in myogenin and FoxO1/O3 stimulated expression of the E3 ubiquitin ligase genes MuRF1 and atrogin1 in the SPF mice treated with metronidazole. Therefore, metronidazole might act as a double-edged sword in skeletal muscle atrophy, causing skeletal muscle neurogenic atrophy through the upregulation of Hdac4 and myogenin and FoxO1/O3-mediated protein degradation, resulting in the observed decrease in larger myofibers and consequent reduced hind limb muscle weight. The effect of metronidazole on skeletal muscle neurogenic atrophy genes in the SPF mice might explain the peripheral neuropathy observed in patients treated with metronidazole. 
We can speculate that the endotoxins produced by metronidazole-resistant proteobacteria might initiate a tumor necrosis factor $\alpha$-mediated inflammatory response, inhibiting Akt signaling and upregulating the FoxO target genes MuRF1, atrogin1, and Pdk4 [36]. Moreover, the upregulation of Pdk4 in SPF mice treated with metronidazole may inactivate the pyruvate dehydrogenase complex, which is rate-limiting in muscle carbohydrate oxidation [37].

Furthermore, our results demonstrate a novel effect of metronidazole on genes involved in the control of muscle circadian rhythm, energy metabolism, and RNA epigenetic modifications. The gut microbiota and its daily rhythmicity of produced metabolites is reported to modulate host circadian chromatin dynamics and transcriptome in intestine and liver [2]. It was proposed that microbiota-generated signals are integrated by peripheral tissues to temporally organize genome-wide transcription [2]. The mammalian circadian clock is a transcriptional and translational negative feedback loop controlled by the core clock gene products Clock, Bmal1, Per1-3, and Cry1 and Cry2, eventually regulating many biological processes [38]. The peripheral clocks in different organs are coordinated by both the central master clock located in the suprachiasmatic nucleus of the hypothalamus and zeitgebers such as light/dark, feeding/fasting, and rest/activity cycles [39]. The skeletal muscle peripheral clock and the clock-controlled genes have been implicated in energy metabolism, myokine secretion, regeneration, and tissue homeostasis [16-18]. Our results show that alteration of the microbiota composition with metronidazole in mice affects the host's circadian rhythm and metabolism of skeletal muscle. Expression levels of the core clock genes Per2 and Cry2 were significantly upregulated in SPF mice treated with metronidazole, with a marginal downregulation in Bmal1, suggesting that the host muscle peripheral clock genes are fine-tuned by the gut microbiota and that dysbiosis affects this regulation process. In GF mice, we observed a microbiota-independent effect of metronidazole on muscle, which interestingly contrasted with the effect seen with microbiota.

The clock-controlled genes Dbp and E4BP4 are critical in determining period lengths in rat-1 fibroblasts and act as positive and negative regulators of transcriptional activity, respectively [40]. Our results show an increase in Dbp expression level that was not significant because of the large error limits among the metronidazole-treated SPF mice as compared to nontreated controls. However, we also found a significant increase in expression level of E4BP4 in the SPF mice treated with metronidazole, although the relevance of this observation remains to be elucidated. Skeletal muscle-specific Bmal1 ablation in mice results in decreased glucose uptake and oxidation, with increased Pdk4 expression causing a switch toward increased lipid metabolism in the muscles of these mice [41]. Our results demonstrate a similar effect with metronidazole treatment in SPF mice, indicating that these mice might undergo increased use of lipids for energy metabolism.

Both adiponectin and PPAR $\gamma$ were significantly upregulated in metronidazole-treated SPF mice. We speculate that these effects might enhance fatty acid uptake and insulin sensitivity of skeletal muscle. Furthermore, FoxO regulates adiponectin signaling through AdipoR, which is critical in regulating lipid and glucose homeostasis and reducing oxidative stress [42].

Our results do not rule out the alternate possibility of changes in the gut mucosal barrier leading to skeletal muscle atrophy because of poor digestion and nutrient absorption, which remains to be investigated. Interestingly, malnutrition in children has been associated with an increase in Proteobacteria [43]. In addition, a limitation of this study is that the tissue samples were harvested in a single time window (zeitgeber time (ZT) 4-6). However, this time point was chosen based on our finding that in GF mice, the strongest alteration in the expression of clock and clock effector genes was observed at ZT6 [19]. Furthermore, it is likely that impaired peripheral nerve activity might be accompanied by muscle incoordination, leading to altered expression of muscle clock genes. Although further studies are required to elucidate the detailed molecular mechanism by which the metronidazole-mediated alteration of gut microbiota composition modulates skeletal muscle chronometabolism, the observed skeletal muscle atrophy is likely the result of disturbed expression of both neurogenic atrophy genes and protein degradation genes. 
To conclude, metronidazole causes gut dysbiosis with increased susceptibility to colonization of Proteobacteria and enrichment of Erysipelotrichales. Importantly, metronidazole causes skeletal muscle atrophy and modulates muscle chronometabolism. These data support a role for the observed side effect of metronidazole-mediated peripheral neuropathy in human patients.

\section{Materials and Methods}

\subsection{Animals}

Wild-type (WT; C57Bl/6J) mice were maintained at the SingHealth Experimental Medicine Centre (SEMC), Singapore. The mice were inbred and housed in the SPF or GF facility of the SEMC. All mice were housed in microisolator units of 5 males per cage and fed ad libitum with food and water. The SPF WT mice were fed a standard irradiated chow diet and the GF mice a standard autoclaved chow diet. All mice were maintained on a 12:12 h light and dark cycle. All mice were euthanized using $\mathrm{CO}_{2}$ gas at ZT 4-6, where ZT0 is 07:30 and ZT12 is 19:30 at the start of the light and dark periods, respectively. The animal work was approved and performed in accordance with the relevant guidelines and regulations of the Institutional Animal Care and Use Committee (IACUC Reference No. 2015/SHS/1133), SingHealth Experimental Medicine Centre, Singapore.

\subsection{Antibiotic Metronidazole Treatment}

Adult male WT SPF mice at 6-7 months of age and GF mice at 2 months of age were administered $1 \mathrm{~g} / \mathrm{L}$ metronidazole (M-840-100; Gold Biotechnology, St. Louis, MO, USA) in drinking water for 4 weeks. Control WT SPF and GF mice received no metronidazole in drinking water. The drinking water was changed twice weekly to compensate for the effects of the half-life of the drug.

\subsection{Mouse Fecal Pellet and Tissue Harvest}

Mouse fecal pellets were collected before euthanization, snap frozen in liquid nitrogen, and stored at $-80^{\circ} \mathrm{C}$ until further analysis of the microbiota composition by $16 \mathrm{~s}$ rRNA gene sequencing. The hind limb tibialis anterior, extensor digitorum longus, gastrocnemius, soleus, and quadriceps muscles were harvested from both legs, weighed, snap frozen in liquid nitrogen, and stored at $-80^{\circ} \mathrm{C}$ until further analysis.

\section{4. $16 S$ rRNA Gene Sequencing of the Fecal Microbiota Population}

Bacterial genomic DNA extraction, amplification, and 16S rRNA gene sequencing of the metronidazole-treated SPF WT mice was carried out using a FastDNA ${ }^{\mathrm{TM}}$ SPIN kit according to the manufacturer's protocol (MP Biomedical, Aurora, CT, USA) by Chunlab Inc., Seoul, South Korea [44]. Briefly, the DNA was first amplified using barcoded primers specific to the V1 and V3 regions of the bacterial 16S rRNA genes. Sequences were then sorted out by their respective unique barcodes, and low-quality reads were removed. Bacterial 16S rRNA gene sequencing of $1 \mu \mathrm{g}$ of PCR amplified product of each sample was done using the 454 GS FLX Titanium Sequencing System (Roche, Branford, FL, USA). Sequence reads were identified using the EZBioCloud Genome Database. Fecal microbial composition, diversity, and clustering were analyzed using the CLcommunity bioinformatics software provided by Chunlab Inc., Seoul, Korea.

\subsection{Histology}

The left hind leg tibialis anterior muscle was OCT (optimum cutting temperature) embedded in liquid nitrogen-cooled isopentane and stored at $-80^{\circ} \mathrm{C}$. Serial transverse cryosections $10 \mu \mathrm{m}$ thick were obtained from the mid-belly region using a Leica cryostat machine. The sections were stained with hematoxylin and eosin, cleared, and mounted in DPX (distyrene plasticiser xylene) mounting compound. Representative pictures were taken with the brightfield $10 \times$ objective of a Leica microscope and analyzed with Image J software (National Institutes of Health, Bethesda, MD, USA). 


\subsection{RNA Isolation and RT-qPCR Analysis}

Total RNA was isolated from the gastrocnemius muscle using Trizol reagent and a PureLink ${ }^{\circledR}$ RNA Mini Kit (Life Technologies, Carlsbad, CA, USA) according to the manufacturer's instructions. cDNA was synthesized from $1 \mu \mathrm{g}$ of RNA using iScript Reverse Transcription Supermix (Bio-Rad, Hercules, CA, USA). Real-time quantitative PCR was performed with KAPA SYBR FAST qPCR kits (KAPA Biosystems, Wilmington, MA, USA). The oligonucleotides (Supplementary Table S1) were ordered from Integrated DNA Technologies, Singapore. The relative mRNA levels were quantified using the ABI StepOne Plus RT-qPCR System in accordance with KAPA Biosystem cycling conditions. All amplified PCR products were calculated based on comparative delta-Ct method and normalized to endogenous glyceraldehyde-3-phosphate dehydrogenase mRNA levels.

\subsection{Statistical Analysis}

Statistical differences between groups were determined using unpaired two-tailed student's $t$-tests, and the results were considered significant at $p<0.05\left(^{*}\right), p<0.01\left({ }^{* *}\right)$, or $p<0.001\left(^{* * *}\right)$. Data are expressed as mean \pm standard error of the mean (SEM).

Supplementary Materials: Supplementary materials can be found at http://www.mdpi.com/1422-0067/19/8/ 2418/s1.

Author Contributions: Conceptualization, R.M. and W.W.; Data curation, R.M. and H.Y.P.O.; Formal analysis, H.Y.P.O. and C.K.T.; Funding acquisition, W.W.; Investigation, R.M., H.Y.P.O., C.K.T. and E.P.; Methodology, R.M., H.Y.P.O., C.K.T. and E.P.; Project administration, R.M. and W.W.; Resources, R.M. and W.W.; Supervision, W.W.; Validation, R.M., H.Y.P.O. and W.W.; Writing-original draft, R.M., H.Y.P.O., C.K.T., E.P. and W.W.; Writing-review \& editing, R.M., H.Y.P.O., C.K.T., E.P. and W.W.

Funding: This work was supported by the startup grant to Lee Kong Chian School of Medicine, Nanyang Technological University, Singapore (WW). H.Y.P.O. was supported by a scholarship and bench fees from Interdisciplinary Graduate School, NTU Institute for Health Technologies, Nanyang Technological University, Singapore.

Acknowledgments: We thank Rachel Tee for her help in manuscript submission.

Conflicts of Interest: All authors declare no conflict of interest.

\section{Abbreviations}

$\begin{array}{ll}\text { Clock } & \text { Circadian locomotor output cycles kaput } \\ \text { Bmal1 } & \text { Brain and muscle ARNT-like 1 } \\ \text { Per } & \text { Period } \\ \text { Cry } & \text { Cryptochrome } \\ \text { Ror } & \text { RAR-related orphan receptor } \\ \text { E4BP4 } & \text { E4 promotor binding protein } 4 \\ \text { Dbp } & \text { D site binding protein } \\ \text { Akt } & \text { Akt serine/threonine kinase } \\ \text { MuRF1 } & \text { Muscle RING-finger protein 1 } \\ \text { MyoD } & \text { Myogenic differentiation } \\ \text { Mul1 } & \text { Mitochondrial E3 ubiquitin protein ligase 1 } \\ \text { LC3-1 } & \text { MAF1 light chain 3-like protein 1 } \\ \text { Ppar } & \text { Peroxisome proliferator activated receptor } \\ \text { Pgc-1 } \alpha & \text { Ppar gamma coactivator 1 alpha } \\ \text { Sirt1 } & \text { Sirtuin 1 } \\ \text { AMPK } & \text { AMP-activated protein kinase } \\ \text { AdipoR } & \text { Adiponectin receptor } \\ \text { Fto } & \text { Fat and obesity-associated protein }\end{array}$




\section{References}

1. Jin, Y.; Wu, Y.; Zeng, Z.; Jin, C.; Wu, S.; Wang, Y.; Fu, Z. From the cover: Exposure to oral antibiotics induces gut microbiota dysbiosis associated with lipid metabolism dysfunction and low-grade inflammation in mice. Toxicol. Sci. 2016, 154, 140-152. [CrossRef] [PubMed]

2. Thaiss, C.A.; Levy, M.; Korem, T.; Dohnalova, L.; Shapiro, H.; Jaitin, D.A.; David, E.; Winter, D.R.; Gur-BenAri, M.; Tatirovsky, E.; et al. Microbiota diurnal rhythmicity programs host transcriptome oscillations. Cell 2016, 167, 1495-1510. [CrossRef] [PubMed]

3. Voigt, R.M.; Forsyth, C.B.; Green, S.J.; Mutlu, E.; Vitaterna, M.H.; Turek, F.W.; Keshavarzian, A. Circadian disorganization alters intestinal microbiota. PLoS ONE 2014, 9, e97500. [CrossRef] [PubMed]

4. Rejinders, D.; Goossens, G.H.; Hermes, G.D.; Neis, E.P.; van der Beek, C.M.; Most, J.; Lenaerts, K.; Kootte, R.S.; Nieuwdorp, M.; Groen, A.K.; et al. Effects of gut microbiota manipulation by antibiotics on host metabolism in obese humans: A randomized double-blind placebo-controlled trial. Cell Metab. 2016, 24, 63-74. [CrossRef] [PubMed]

5. Shoaie, S.; Ghaffari, P.; Kovatcheva-Datchar, P.; Mardinoglu, A.; Sen, P.; Pujos-Guillot, E.; de Wouters, T.; Juste, C.; Rizkalla, S.; Chilloux, J.; et al. Quantifying diet-induced metabolic changes of the human gut microbiome. Cell Metab. 2015, 22, 320-331. [CrossRef] [PubMed]

6. Fujisaka, S.; Ussar, S.; Clish, C.; Devkota, S.; Dreyfuss, J.M.; Sakaguchi, M.; Soto, M.; Konishi, M.; Softic, S.; Altindis, E.; et al. Antibiotic effects on gut microbiota and metabolism are host dependent. J. Clin. Investig. 2016, 126, 4430-4443. [CrossRef] [PubMed]

7. Abeles, S.R.; Jones, M.B.; Santiago-Rodriguez, T.M.; Ly, M.; Klitgord, N.; Yooseph, S.; Nelson, K.E.; Pride, D.T. Microbial diversity in individuals and their household contacts following typical antibiotic courses. Microbiome 2016, 4, 39. [CrossRef] [PubMed]

8. Morgun, A.; Dzutsev, A.; Dong, X.; Greer, R.L.; Sexton, D.J.; Ravel, J.; Schuster, M.; Hsiao, W.; Matzinger, P.; Shulzhenko, N. Uncovering effects of antibiotics on the host and microbiota using transkingdom gene networks. Gut 2015, 64, 1732-1743. [CrossRef] [PubMed]

9. Rakoff-Nahoum, S.; Paglino, J.; Eslami-Varzaneh, F.; Edberg, S.; Medzhitov, R. Recognition of commensal microflora by toll-like receptors is required for intestinal homeostasis. Cell 2004, 118, 229-241. [CrossRef] [PubMed]

10. Backhed, F.; Ding, H.; Wang, T.; Hooper, L.V.; Koh, G.Y.; Nag, A.; Semenkovich, C.F.; Gordon, J.I. The gut microbiota as an environmental factor that regulates fat storage. Proc. Natl. Acad. Sci. USA 2004, 101, 15718-15723. [CrossRef] [PubMed]

11. Turnbaugh, P.J.; Ruth, E.L.; Michael, A.M.; Vincent, M.; Elaine, R.M.; Jeffrey, I.G. An obesity-associated gut microbiome with increased capacity for energy harvest. Nature 2006, 444, 1027-1031. [CrossRef] [PubMed]

12. Vijay-kumar, M.; Aitken, J.D.; Carvalho, F.A.; Cullender, T.C.; Mwangi, S.; Srinivasan, S.; Sitaraman, S.V.; Knight, R.; Le, R.E.; Gewirtz, A.T. Metabolic syndrome and altered gut microbiota in mice lacking Toll-like receptor 5. Science 2010, 328, 228-231. [CrossRef] [PubMed]

13. Lamp, K.C.; Freeman, C.D.; Klutman, N.E.; Lacy, M.K. Pharmacokinetics and pharmacodynamics of the nitroimidazole antimicrobials. Clin. Pharmacokinet. 1999, 36, 353-373. [CrossRef] [PubMed]

14. Lewis, B.B.; Buffie, C.G.; Carter, R.A.; Leiner, I.; Toussaint, N.C.; Miller, L.C.; Gobourne, A.; Ling, L.; Pamer, E.G. Loss of microbiota-mediated colonization resistance to Clostridium difficile infection with oral vancomycin compared with metronidazole. J. Infect. Dis. 2015, 212, 1656-1665. [CrossRef] [PubMed]

15. Bhattacharyya, S.; Darby, R.R.; Raibagkar, P.; Gonzalez Castro, L.N.; Berkowitz, A.L. Antibiotic-associated encephalopathy. Neurology 2016, 86, 963-971. [CrossRef] [PubMed]

16. Andrews, J.L.; Zhang, X.; McCarthy, J.J.; McDearmon, E.L.; Hornberger, T.A.; Russell, B.; Campbell, K.S.; Arbogast, S.; Reid, M.B.; Walker, J.R.; et al. Clock and Bmal1 regulate MyoD and are necessary for maintenance of skeletal muscle phenotype and function. Proc. Natl. Acad. Sci. USA 2010, 107, 19090-19095. [CrossRef] [PubMed]

17. Harfmann, B.D.; Elizabeth, A.S.; Maureen, T.K.; Brian, A.H.; Xiping, Z.; Karyn, A.E. Muscle-specific loss of Bmal1 leads to disrupted tissue glucose metabolism and systemic glucose homeostasis. Skelet. Muscle 2016, 6, 12. [CrossRef] [PubMed] 
18. Perrin, L.; Loizides-Mangold, U.; Skarupelova, S.; Pulimeno, P.; Chanon, S.; Robert, M.; Bouzakri, K.; Modoux, C.; Roux-Lombard, P.; Vidal, H.; et al. Human skeletal myotubes display a cell-autonomous circadian clock implicated in basal myokine secretion. Mol. Metab. 2015, 4, 834-845. [CrossRef] [PubMed]

19. Montagner, A.; Korecka, A.; Polizzi, A.; Lippi, Y.; Blum, Y.; Canlet, C.; Trembla-Franco, M.; Gautier-Stein, A.; Burcelin, R.; Yen, Y.C.; et al. Hepatic circadian clock oscillators and nuclear receptors integrate microbiome-derived signals. Sci. Rep. 2016, 6, 20127. [CrossRef] [PubMed]

20. Moresi, V.; Williams, A.H.; Meadows, E.; Flynn, J.M.; Potthoff, M.J.; McAnally, J.; Shelton, J.M.; Backs, J.; Klein, W.H.; Richardson, J.A.; et al. Myogenin and class II HDACs control neurogenic muscle atrophy by inducing E3 ubiquitin ligases. Cell 2010, 143, 35-45. [CrossRef] [PubMed]

21. Mammucari, C.; Milan, G.; Romanello, V.; Masiero, E.; Rudolf, R.; Del Piccolo, P.; Burden, S.J.; Di Lisi, R.; Sandri, C.; Zhao, J.; et al. FoxO3 controls autophagy in skeletal muscle in vivo. Cell Metab. 2007, 6, 458-471. [CrossRef] [PubMed]

22. Sandri, M.; Sandri, C.; Gilbert, A.; Skurk, C.; Calabria, E.; Picard, A.; Walsh, K.; Schiaffino, S.; Lecker, S.H.; Goldberg, A.L. FoxO transcription factors induce the atrophy-related ubiquitin ligase atrogin-1 and cause skeletal muscle atrophy. Cell 2004, 117, 399-412. [CrossRef]

23. Furuyama, T.; Kitayama, K.; Yamashita, H.; Mori, N. Forkhead transcription factor FOXO1, (FKHR)-dependent induction of PDK4 gene expression in skeletal muscle during energy deprivation. Biochem. J. 2003, 375, 365-371. [CrossRef] [PubMed]

24. Kamei, Y.; Miura, S.; Suzuki, M.; Kai, Y.; Mizukami, J.; Taniguchi, T.; Mochida, K.; Hata, T.; Matsuda, J.; Aburatani, H.; et al. Skeletal muscle FOXO1 (FKHR) transgenic mice have less skeletal muscle mass, down-regulated Type 1 (slow twitch/red muscle) fiber genes, and impaired glycemic control. J. Biol. Chem. 2004, 279, 41114-41123. [CrossRef] [PubMed]

25. Miyamoto, Y.; Sancar, A. Circadian regulation of cryptochrome genes in the mouse. Brain Res. Mol. Brain Res. 1999, 71, 238-243. [CrossRef]

26. Koyanagi, S.; Okazawa, S.; Kuramoto, Y.; Ushijima, K.; Shimeno, H.; Soeda, S.; Okamura, H.; Ohdo, S. Chronic treatment with prednisolone represses the circadian oscillation of clock gene expression in mouse peripheral tissue. Mol. Endocrinol. 2006, 20, 573-583. [CrossRef] [PubMed]

27. Sauermann, R.; Schmidt, W.M.; Krebs, M.; Brunner, M.; Muller, M. Ramipril modulates circadian gene expression in skeletal muscle. Pharmacogenet. Genom. 2011, 21, 751-759. [CrossRef] [PubMed]

28. Barnea, M.; Madar, Z.; Froy, O. High-fat diet followed by fasting disrupts circadian expression of adiponectin signaling pathway in muscle and adipose tissue. Obesity 2010, 18, 230-238. [CrossRef] [PubMed]

29. Hashinaga, T.; Wada, N.; Otabe, S.; Yuan, X.; Kurita, Y.; Kakino, S.; Tanaka, K.; Sato, T.; Kojima, M.; Ohki, T.; et al. Modulation by adiponectin of circadian clock rhythmicity in model mice for metabolic syndrome. Endocr. J. 2013, 60, 483-492. [CrossRef] [PubMed]

30. Liao, S.; Sun, H.; Xu, C. YTH Domain: A Family of $\mathrm{N}^{6}$-methyladenosine $\left(\mathrm{m}^{6} \mathrm{~A}\right)$ Readers. Genom. Proteom. Bioinform. 2018, 16, 99-107. [CrossRef] [PubMed]

31. Batista, P.J. The RNA Modification $\mathrm{N}^{6}$-methyladenosine and Its Implications in Human Disease. Genom. Proteom. Bioinform. 2017, 15, 154-163. [CrossRef] [PubMed]

32. Kudou, K.; Komatsu, T.; Nogami, J.; Maehara, K.; Harada, A.; Saeki, H.; Oki, E.; Maehara, Y.; Ohkawa, Y. The requirement of Mettl3-promoted MyoD mRNA maintenance in proliferative myoblasts for skeletal muscle differentiation. Open Biol. 2017, 7, 170119. [CrossRef] [PubMed]

33. Chen, Y.J.; Wu, H.; Wu, S.D.; Lu, N.; Wang, Y.T.; Liu, H.N.; Dong, L.; Liu, T.T.; Shen, X.Z. Parasutterella, in association with irritable bowel syndrome and intestinal chronic inflammation. J. Gasteroenterol. Hepatol. 2018. [CrossRef] [PubMed]

34. Ticinesi, A.; Lauretani, F.; Milani, C.; Nouvenne, A.; Tana, C.; Del Rio, D.; Maggio, M.; Ventura, M.; Meschi, T. Aging gut microbiota at the cross-road between nutrition, physical fraility, and sarcopenia: Is there a gut-muscle axis? Nutrients 2017, 9, 1303. [CrossRef] [PubMed]

35. Kim, K.A.; Jeong, J.J.; Yoo, S.Y.; Kim, D.H. Gut microbiota lipopolysaccharide accelerates inflamm-aging in mice. BMC Microbiol. 2016, 16, 9. [CrossRef] [PubMed]

36. Crossland, H.; Constantin-Teodosiu, D.; Gardiner, S.M.; Constantin, D.; Greenhaff, P.L. A potential role for Akt/FOXO signalling in both protein loss and the impairment of muscle carbohydrate oxidation during sepsis in rodent skeletal muscle. J. Physiol. 2008, 586, 5589-5600. [CrossRef] [PubMed] 
37. Manickam, R.; Wahli, W. Roles of Peroxisome Proliferator-Activated Receptor $\beta / \delta$ in skeletal muscle physiology. Biochimie 2017, 136, 42-48. [CrossRef] [PubMed]

38. McCarthy, J.J.; Andrews, J.L.; McDearmon, E.L.; Campbell, K.; Barber, B.K.; Miller, B.H.; Walker, J.R.; Hogenesh, J.B.; Takahashi, J.S.; Esser, K.A. Identification of the circadian transcriptome in adult mouse skeletal muscle. Physiol. Genom. 2007, 31, 86-95. [CrossRef] [PubMed]

39. Bae, K.; Kisoo, L.; Younguk, S.; Haesang, L.; Dongyong, K.; Inbo, C. Differential effects of two period genes on the physiology and proteomic profiles of mouse anterior tibialis muscles. Mol. Cells 2006, 22, $275-284$. [PubMed]

40. Yamajuku, D.; Shibata, Y.; Kitazawa, M.; Katakura, T.; Urata, H.; Kojima, T.; Takayasu, S.; Nakata, O.; Hashimoto, S. Cellular DBP and E4BP4 proteins are critical for determining the period length of the circadian oscillator. FEBS Lett. 2011, 585, 2217-2222. [CrossRef] [PubMed]

41. Dyar, K.A.; Ciciliot, S.; Wright, L.E.; Bienso, R.S.; Tagliazucchi, G.M.; Patel, V.R.; Forcato, M.; Paz, M.I.; Gudiksen, A.; Solagna, F.; et al. Muscle insulin sensitivity and glucose metabolism are controlled by the intrinsic muscle clock. Mol. Metab. 2013, 3, 29-41. [CrossRef] [PubMed]

42. Park, H.S.; Lim, J.H.; Kim, M.Y.; Hong, Y.A.; Choi, S.R.; Chung, S.; Kim, H.W.; Choi, B.S.; Kim, Y.S.; Chang, Y.S.; et al. Resveratrol increases AdipoR1 and AdipoR2 expression in type 2 diabetic nephropathy. J. Transl. Med. 2016, 14, 176. [CrossRef] [PubMed]

43. Monira, S.; Nakamura, S.; Gotoh, K.; Izutsu, K.; Watanabe, H.; Alam, N.H.; Endtz, H.P.; Cravioto, A.; Ali, SI.; Nakaya, T.; et al. Gut microbiota of healthy and malnourished children in Bangladesh. Front. Microbiol. 2011, 2, 228. [CrossRef] [PubMed]

44. Chun, J.; Kim, K.Y.; Lee, J.H.; Choi, Y. The analysis of oral microbial communities of wild-type and toll-like receptor 2-deficient mice using a 454 GS FLX Titanium pyrosequencer. BMC Microbiol. 2010, 10, 101. [CrossRef] [PubMed]

(C) 2018 by the authors. Licensee MDPI, Basel, Switzerland. This article is an open access article distributed under the terms and conditions of the Creative Commons Attribution (CC BY) license (http:/ / creativecommons.org/licenses/by/4.0/). 